\title{
MOBILE 3D MAPPING WITH A LOW-COST UAV SYSTEM
}

\author{
F. Neitzel ${ }^{\text {a, }}$, J. Klonowski ${ }^{\text {b }}$ \\ ${ }^{a}$ TU Berlin, Department of Geodesy and Geoinformation Science, 10623 Berlin, Germany - frank.neitzel@tu-berlin.de \\ b i3mainz - Institute for Spatial Information and Surveying Technology, FH Mainz, 55128 Mainz - joerg.klonowski@fh- \\ mainz.de
}

Commission I, WG I/V

KEY WORDS: Mobile Mapping, Unmanned Aerial Vehicle, UAV, Photogrammetry, 3D Point cloud, Flight planning

\begin{abstract}
:
In this contribution it is shown how an UAV system can be built at low costs. The components of the system, the equipment as well as the control software are presented. Furthermore an implemented programme for photogrammetric flight planning and its execution are described. The main focus of this contribution is on the generation of 3D point clouds from digital imagery. For this web services and free software solutions are presented which automatically generate 3D point clouds from arbitrary image configurations. Possibilities of georeferencing are described whereas the achieved accuracy has been determined. The presented workflow is finally used for the acquisition of 3D geodata. On the example of a landfill survey it is shown that marketable products can be derived using a low-cost UAV.
\end{abstract}

\section{INTRODUCTION}

Mobile Mapping can be defined as the acquisition of spatiotemporal phenomena by utilising a mobile multi-sensor platform. Its aim is to derive structured object information from the registered data. This information can be handed to a user in form of e.g. maps or digital terrain models (DTM). The process chain in Mobile Mapping consists of the following steps:

1. Mobilisation of the platform,

2. data acquisition,

3. data processing,

4. extraction of object information,

5. allocation of object information to a user.

In case that object information has to be provided immediately the term Rapid Mapping is used. Multi-sensor platforms are being offered by many manufacturers for several years that are equipped, among other parts, with the following components:

- Integrated navigation unit consisting of GNSS receivers (GPS) and an inertial measurement unit (IMU),

- optical 3D measurement system mostly represented through one or several laser scanners and cameras.

Such systems, whose acquisition costs start at approximately 100000 Euro, can be adapted at planes, helicopters or land crafts. A field of application for terrestrial systems is for instance the acquisition of 3D city models, whereas aerial systems cover e.g. surveys of open cast mining. Aerial applications are as a rule quite cost-intensive which is mainly caused by the expenses for the flight itself.

Areas of small-sized occurrence are suitable for geodata acquisition by applying Unmanned Aerial Vehicles (UAV), as these platforms are cost-effective as well as flexible and bridge the gap between terrestrial and aerial data collection. If the aircraft is equipped with a camera in order to carry out photogrammetric surveys the term "UAV-Photogrammetry" should be used. A sound disquisition referring to this topic is given by Eisenbeiß (2009), who also analysed the capability of several software packages for processing of aerial photographs taken from UAVs. Eisenbeiß (2009, p. 171) concludes that not all tested software packages are capable of solving the entire range of problems that arise from UAV-Photogrammetry. He also deduces that this limitation is caused by the fact that these programmes have been developed for standard aerial imagery and not for arbitrary configurations.

Meanwhile a selection of capable web services and software packages at no charge are available that are applicable for unordered imagery. In this contribution chances for geodata acquisition by applying the above mentioned technique in combination with UAVs are pointed out.

\section{ASSEMBLY OF THE UAV SYSTEM}

The applied UAV has been provided as an assembly set by HiSystems GmbH. Based on its equipment the model MK Okto with eight propellers has been chosen, which is also referred to as Oktokopter. The order of the kit has been placed at www.mikrokopter.de where construction manuals are provided as well. The following sections present all employed components.

\subsection{Components}

A frame, which consists of aluminium square tubes and carbon fibre base plates, describes the basis of the system. The landing gear is made of plastic. The system is powered by Roxxy 282735 brushless motors that drive right and left rotating EPP1045 propellers. Brushless Control V1.2 sets the rotary speed of each motor separately.

A Flight Control circuit board controls sensor fusion and current flight information by applying gyroscopes, accelerometers and an air pressure sensor. Information about an

\footnotetext{
* Corresponding author
} 
intended air lane for autonomous flights can be exchanged by a transmitter or other interfaces which are organised by the Navi Control board. Furthermore this component gathers information from the magnetic compass MK3Mag and the GPS module MK-GPS which are then analysed in a set-actual comparison in order to send control commands to the Flight Control module. A MicroSD card reader on the Navi Control board is able to record details about a flight. The magnetic compass MK3Mag is used to stabilise current positioning and directional referencing when navigating to set waypoints. A passive ublox-LEA4H GPS antenna is equipped on the MK-GPS board.

\subsection{Equipment}

A Spektrum DX7 remote control sends commands within the $2.4 \mathrm{GHz}$ frequency band that subsequently is processed by the Flight Control module. A bidirectional solution that works within $2.4 \mathrm{GHz}$ as well is provided by a serial F2M03GXA Bluetooth interface. By applying an identical interface on a personal computer an increase in coverage can be obtained. A video signal in analogue format can be broadcasted via $\mathrm{A} / \mathrm{V}$ transmitter in a frequency range of $5.8 \mathrm{GHz}$.

A MK HiSight II camera mount applies a servomotor that allows stageless displacement of pitch angles and actively compensates for its variations whereas a damping system adjusts for occurring rolling moments.

Furthermore a laptop that is suitable for outdoor use that is equipped with a Windows operating system is needed to execute necessary programmes for the flight control, which are described in the next section. Also the laptop should possess over an integrated Bluetooth module with high range to establish a connection to the Oktokopter.

\subsection{Software}

In order to operate the UAV the MikroKopter Tool programme is used. Several visualisation and analysis tools of flight data are provided via a graphical user interface as well as options for waypoint or trajectory pursuit modes. The programme version $1.70 \mathrm{a}$ was used and is downloadable at no charge under www.mikrokopter.de/ucwiki/MikroKopterTool.

\subsection{Camera}

A digital camera should be applied to capture imagery off the UAV. To maximise time of flight a lightweight Canon Digital Ixus 100 IS compact camera has been chosen. The total weight in combination with the MK HiSight II camera mount adds up to ca. $260 \mathrm{~g}$. The telling argument to choose the Canon Ixus camera is the option to run adaptable scripts by using the CHDK (Canon Hack Development Kit) software which is available at no charge under http://chdk.wikia.com/wiki/CHDK. While running the camera on board the UAV a script has been used that takes an arbitrary number of images with a defined time interval.

\subsection{Technical Data and Costs}

The UAV system with all described components is shown in Figure 1. The technical key data of the system are:

- Diameter:

ca. $1 \mathrm{~m}$

- Net weight:

$1.2 \mathrm{~kg}$

- Drivetrain:
- Power supply:

Lithium-Polymer Accumulator (5000 mAh, $14.8 \mathrm{~V})$

- Take-off weight: $2 \mathrm{~kg}$ (with camera)

- Maximum altitude:

- Flight time:

$350 \mathrm{~m}$ ca. 20 min (with camera)

The overall cost for the UAV system excluding the laptop adds up to roughly 3000 Euro.

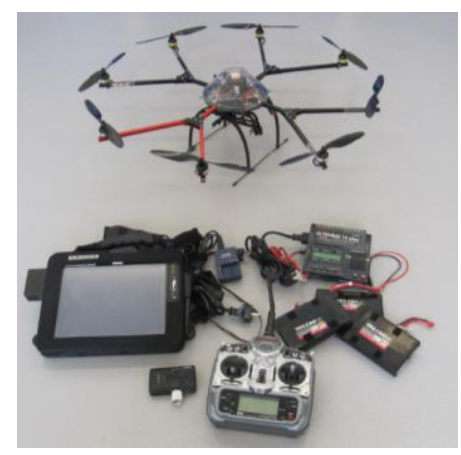

Figure 1. UAV with equipment and camera

\section{FLIGHT PLANNING AND AERIAL SURVEY}

\subsection{Flight Planning}

In order to generate 3D information from aerial photographs a flight plan has to be derived. As the in section 2.3 mentioned MikroKopter Tool is only able to manually set single waypoints it can't be used for planning purposes. Thus a Flight Planning Tool has been developed. After selecting the area of interest in Google Earth a polygon covering the region will be written to a KML file which is then transferred to the implemented programme. After defining flying altitude, longitudinal and transversal coverage coordinates for all waypoints are computed and transferred as a WPL file into MikroKopter Tool. As the deployed version of the programme is only able to manage 12 waypoints larger files have to be split in several files.

\subsection{Aerial Survey}

In order to set up the aerial survey the MikroKopter software has got to be started whereas the first waypoint file is transferred to the UAV. The camera is then configured by applying a CHDK script in order to capture multiple images. The interval in-between images has been set to 2 seconds so that at least two pictures will be captured at every waypoint. Furthermore the autofocus is disabled after the focus has been adjusted at the first waypoint in order to keep a suitable setting for the complete series of images. This process step finalises the mobilisation of the UAV system.

Subsequently the UAV is started. After reaching the predefined altitude, flight direction is anew controlled and if necessary corrected. Subsequently the UAV is set to GPS waypoint mode in order to head for the first loaded waypoints. Tests have shown that flying the UAV between $3-5 \mathrm{~m} / \mathrm{s}$ breezes is feasible without a problem. Wind speeds above ca. $8 \mathrm{~m} / \mathrm{s}$ led to distinct differences to preset waypoint coordinates. After having successfully flown to all waypoints the "Coming Home" function is activated and landing can be prepared. This process step finalises the data acquisition. 


\section{GENERATION OF 3D POINT CLOUDS}

\subsection{Selection of Adequate Imagery}

The first step of data processing starts with selecting adequate images for further use. During this step, which up to now is carried out manually, images are rejected from further processing that have the following properties:

- Images that have been taken during take-off and landing,

- images that are blurry, under- or overexposed,

- images that do not cover the area of interest,

Experience has shown that $20 \%$ to $40 \%$ have to be eliminated in practice. The remaining images are then used to generate a $3 \mathrm{D}$ point cloud.

\subsection{Software}

This section presents web services and software that automatically generate $3 \mathrm{D}$ point clouds from arbitrary image configurations. For the computation of point clouds Exif metadata provides information on image size and applied focal length of the camera. By automatically extracting features such as contours, edges and feature points, which then have to be allocated in order to describe homologous areas, interior and exterior orientation are computed in a bundle adjustment. During this adjustment calibration parameters of the camera are estimated. An extensive recapitulation of the entire procedure to generate 3D point clouds can be found in (Snavely et al. 2007)

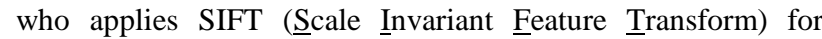
keypoint detection as introduced by Lowe (2004). (Furukawa et al. 2010) propose a multi-stereo-view approach for large unorganised datasets whereas (Furukawa \& Ponce 2010) present a novel algorithm which is based on computation of rectangular patches in overlapping areas of adjacent images.

Microsoft Photosynth is a web service which is accessible under http://photosynth.net at no charge. In order to compute spatial orientations the programme sifts for feature points that subsequently could be used as tie points. As a result of this computationally intensive process 3D coordinates for each matched feature point are calculated whereas interior and exterior orientation parameters for each image are determined. By using the SynthExport programme, which can be found under http://synthexport.codeplex.com, information about the calibration parameters of the camera as well as a description on the exterior orientation and finally the point cloud itself can be exported. A major drawback has to be mentioned which is the need of transmitting data to a web service and that the density of generated point clouds is quite coarse.

ARC3D (Automatic Reconstruction Conduit) is also a free web service for generating $3 \mathrm{D}$ point clouds and meshed surface models from arbitrary imagery, which can be accessed under www.arc3d.be. This service is part of the EPOCH network (European Network of Excellence in Open Cultural Heritage), see www.epoch-net.org for details. In order to transmit digital images to the web service a software has to be installed onto a local computer. After the computation of a project a notification will be send to the user that the results are downloadable. For visualisation purposes the V3D format is used wherefore a suitable viewer is needed where the MeshLab software can be applied. It can be downloaded at no charge under http://meshlab.sourceforge.net. Again the user has to send data to a web service when applying ARC3D, which can be seen as a downside. Generated point clouds feature a high density.
Bundler is a free programme for local data processing on a personal computer, see http://phototour.cs.washington.edu/ bundler for details. The programme generates 3D point clouds from images with an unordered configuration. As a result parameters for calibration and orientation can be accessed as well as a coloured point cloud in PLY file format. An advantage of the programme is that all input data remains in the hands of the user due to the local applicability of the programme. The density of the generated point clouds meets approximately Photosynth whereas details mostly aren't perceptible. The results from Bundler can be used as an input file for the programmes CMVS and PMVS2 which leads to a densification of the computed dataset.

CMVS/PMVS2: PMVS2 is an acronym for Patch-based Multiview Stereo Software Version $\underline{2}$, downloadable at no cost under http://grail.cs.washington.edu/software/pmvs, which is a programme to derive dense, three dimensional point clouds from rectified and oriented images. Files derived with Bundler can be used as input data for this programme. PMVS2 is free software for data processing on a user's local computer. Another aim of the University of Washington who developed these solutions was to rapidly process projects with large amounts of images. The implementation of this ambition can be found in a programme called CMVS which stands for Clustering Views for

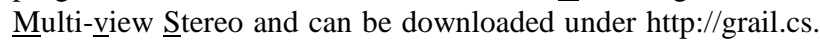
washington.edu/software/cmvs. CMVS performs a clustering process prior to executing PMVS2 which breaks large objects down into smaller entities that are then computed separately. The advantages of these solutions lie within a transparent computation and many possible settings that influence respectively control the result. In comparison to results generated in Bundler and Photosynth a drastic increase in point density can be achieved which leads to a more detailed description of captured objects.

AgiSoft PhotoScan is a commercial product which can be purchased via its developing company AgiSoft and is available for \$ 179 USD in a standard edition whereas \$ 3499 USD have to be invested for the professional version. The following results have been processed by applying the standard edition. Further information on these products can be found under www.agisoft.ru. This programme is executable under Windows operating systems and generates 3D point clouds from arbitrary digital imagery. All data remains with the user as this software can be operated on a local personal computer. The generated point clouds feature a high density which makes details easily recognisable. For the computation of large projects (starting from 100 images upwards) it is recommended to employ a 64bit operating system with at least $6 \mathrm{~GB}$ of RAM. Problems occurred while using a 32bit system where projects couldn't be computed.

\subsection{Comparison of Generated Point Clouds}

In order to evaluate and compare all generated results a parking lot has been surveyed by applying the UAV. After conducting the aerial survey and manually sorting the imagery 99 pictures have been used to compute 3D point clouds by using all presented software packages. Point density and completeness (coverage of the area of interest) have been introduced as quality criteria. A longitudinal overlap of $70 \%$ and a lateral overlap of $60 \%$ have been adopted while flying at $50 \mathrm{~m}$ altitude. The image resolution of all captured JPEG stored pictures has been reduced from 12 to 3 megapixels in order to obtain lower computational costs. It has to be noted that the ARC3D web 
service only reached coverage of $50 \%$ where $3 \mathrm{D}$ information could be computed. The total amount of generated points and hence resulting point density are listed in Table 1 . Note that the column that features the amount of points per $\mathrm{m}^{2}$ has been set into relation to the actual generated expanse. It can be noticed that Photosynth as well as Bundler and PMVS as well as Photoscan generate similar results in terms of point density and point count. ARC3D computes the highest point density.

Table 1. Comparison of generated point clouds

\begin{tabular}{|c|c|c|}
\hline Software & $\begin{array}{c}\text { Total amount of } \\
\text { points }\end{array}$ & Points per $\mathrm{m}^{2}$ \\
\hline Photosynth & 128535 & $\sim 7$ \\
Bundler & 125989 & $\sim 8$ \\
PMVS2 & 1.4 million & $\sim 90$ \\
PhotoScan & 1.3 million & $\sim 110$ \\
ARC3D & 20 million & $\sim 3000$ \\
\hline
\end{tabular}

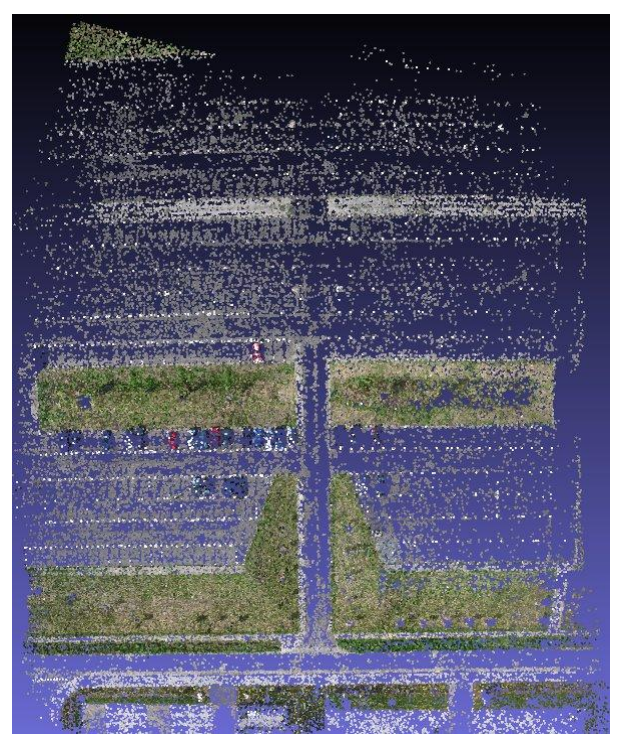

In order to evaluate the coverage of the captured area the derived point clouds are shown in Figure 2 and Figure 3. When comparing the datasets derived from Photosynth and Bundler it becomes obvious that both results share similar characteristics. Both point clouds are patchy in such a way that for instance only the outlines of parking cars have been detected. Datasets derived with PMVS2 and Photoscan both show a considerably higher coverage of the area of interest whereat the point cloud computed with Photoscan doesn't have any gaps within its dataset while PMVS2 created point clouds with smaller holes. ARC3D was only able to generate a point cloud with $50 \%$ of coverage which indeed featured very high point density but nevertheless showed larger gaps of the captured area. A valuable feature for quality assurance is implemented in this software which is a colour coded representation of the 3D point quality.

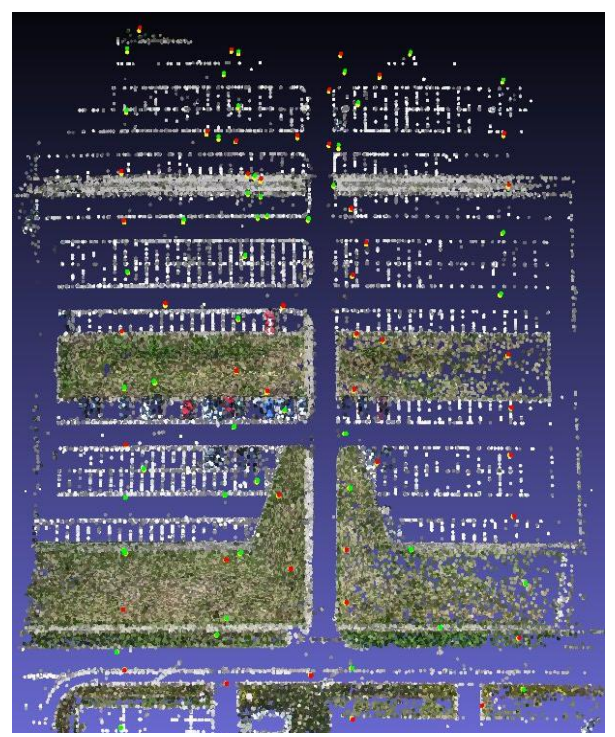

Figure 2. Point cloud generated with Photosynth (left) and Bundler (right)
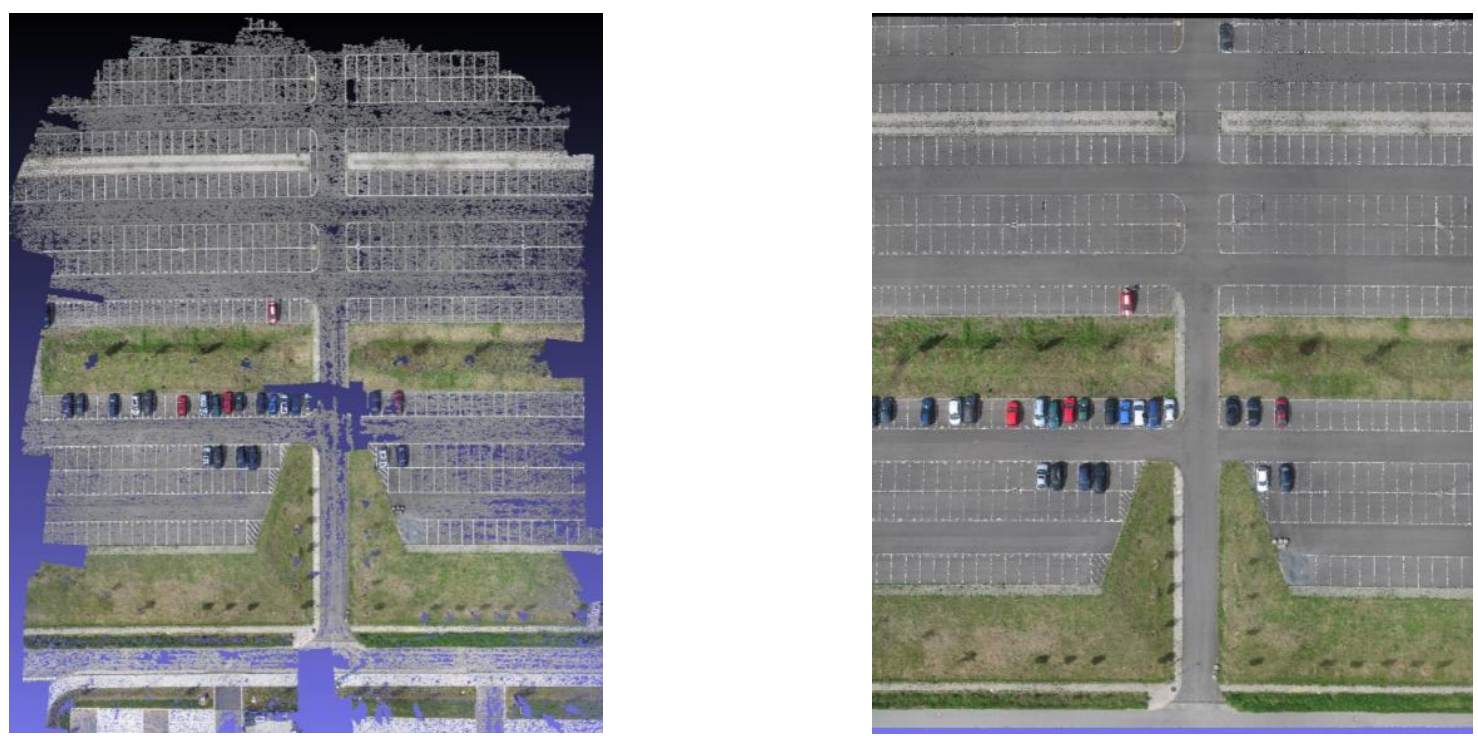

Figure 3. Point cloud computed with PMVS2 (left) and PhotoScan (right) 


\section{GEOREFERENCING}

\subsection{Direct and Indirect Georeferencing}

Direct georeferencing offers the great advantage that no signalisation, no local survey of reference points in the field as well as no determination within the point cloud is needed. Direct georeferencing can be achieved by using data from GPS data that's been recorded during flight. The flight data of the Oktokopter is written to a memory card in a certain interval whereas every entry contains a timestamp in GPS time. Synchronisation between the internal camera clock with regards to GPS time is by then achieved manually.

By means of applying the GeoSetter freeware, which can be downloaded under www.geosetter.de, corresponding coordinates and heights can be integrated into the Exif header of every image. In case that coordinates of one point in time are not available interpolation is conducted. Hence three dimensional Cartesian coordinates in WGS84, which is the superior coordinate system, for all camera positions are at hand. Corresponding Cartesian 3D coordinates of the local system can be derived for instance with Photosynth. By applying a 3D Helmert transformation a transition from local to superior coordinates can be described, which can be achieved by using the Trans3D software, which can be downloaded at no charge under www.xdesy.de.

For the research in this contribution indirect georeferencing has been applied by using six control points that were marginally located within the area of interest. These points have been signalised with checkerboard targets with a size of $25 \mathrm{~cm}$ each and then surveyed by GNSS and SAPOS (German DGNSS Reference Station System). After the aerial survey has been carried out the placed targets had to be detected within the generated point cloud. This step couldn't be fulfilled in the datasets from Photosynth and Bundler due to lack of density. As ARC3D was only able to model half of the test area only results from two solutions, PMVS2 and PhotoScan, were taken into further consideration.

Local coordinates have been determined by using a software called CloudCompare, see www.danielgm.net/cc. The centres of all checkerboards have been sighted with the cursor and all corresponding coordinates have been stored in a list. After all local coordinates have been determined a 3D Helmert transformation has been carried out by using the Trans3D software. By applying these computed parameters a transformation into the superior coordinate system can be applied.

\subsection{Accuracy Analysis}

In order to draw conclusions in terms of absolute accuracy of the generated point clouds all corners of marked parking spots have been surveyed in height and position by applying tacheometry. The following results have been derived with PMVS2 and Photoscan. After determining all coordinates of reference and control points by using CloudCompare a set of transformation parameters has been derived with Trans3D while taking previously surveyed reference points into account. Subsequently all points have been transformed into the superior system. Finally deviations between transformed and geodetically surveyed coordinates have been calculated.

For the results derived with PMVS2 a positional deviation of $235 \mathrm{~mm}$ for the complete test area and $136 \mathrm{~mm}$ within the reference points cluster have been determined on average. The corresponding values for points computed with PhotoScan are $256 \mathrm{~mm}$ respectively $56 \mathrm{~mm}$. The mean deviation in height for points generated with PMVS2 was $5 \mathrm{~mm}$ for the whole test area while $2 \mathrm{~mm}$ were detected within the reference point cluster. The corresponding deviations for results computed with PhotoScan amount to $-5 \mathrm{~mm}$ and $-25 \mathrm{~mm}$.

The characteristics of residuals are presented in Figure 4 and Figure 5, where deviations in position are depicted as vectors, deviations in height as circles and reference points as crosses. These illustrations have been generated with Quantum GIS, an open source GIS, which can be accessed under www.qgis.org. An assimilable characteristic becomes obvious after comparing the vectors in Figure 4 and Figure 5. Their lengths increase from the centre of the point cloud outwards which affirms a rule in geodesy to always place reference points beyond the area of interest to avoid extrapolatory effects.

Height differences from points derived with PMVS2, see Figure 4 , increases from the centre of the dataset notably to the left image boarder. A different pattern emerges from point clouds processed with PhotoScan in Figure 5. Height differences appear to be smaller on the outer boundaries of the dataset whereas a bulging effect towards the centre becomes noticeable. As both datasets have been derived from identical imagery it can be noticed that the results are dependent from the applied software. It also has to be mentioned that the results are dependent on the topography of the test area which leads to the conclusion that no general predications in terms of accuracy can be stated.

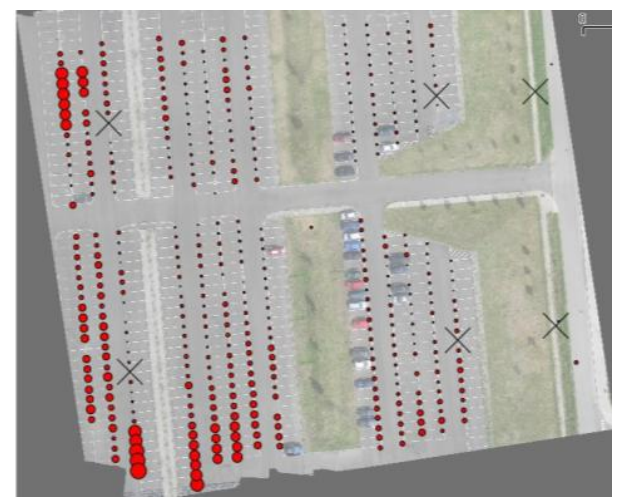

Figure 4. Positional (left) and height deviations (right) of the point cloud derived with PMVS2 

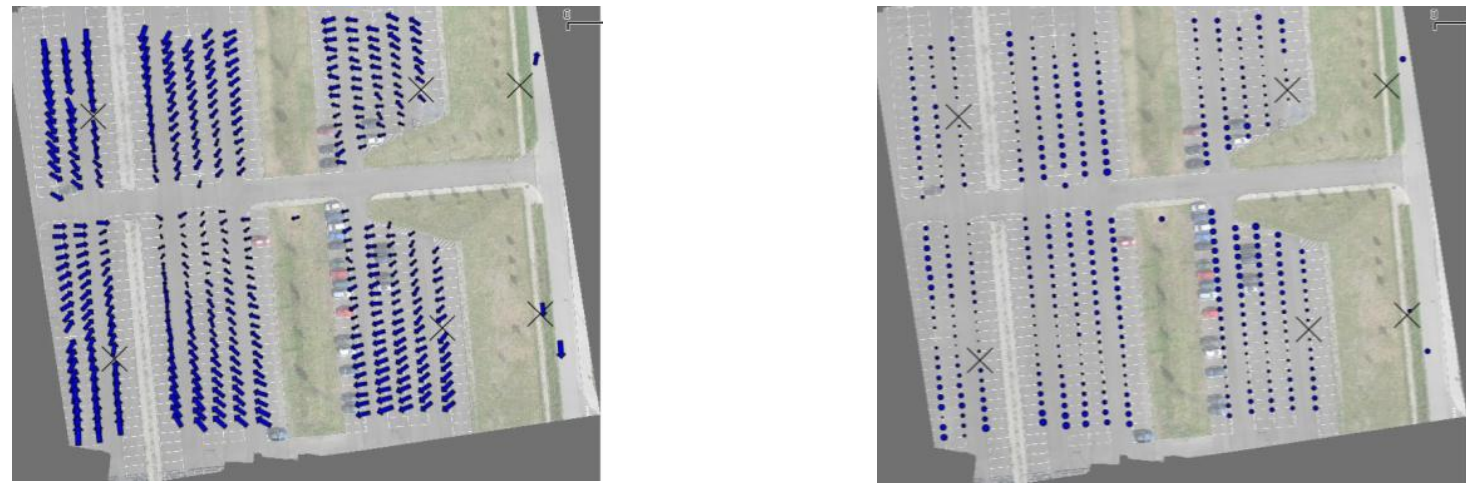

Figure 5. Positional (left) and height deviations (right) of the point cloud computed with PhotoScan

\section{SAMPLE APPLICATION ON A LANDFILL}

Surveying landfills is carried out in order to determine its volume or quantity take-off. The basic principle is built on a continuous registration of the surface within the landfill and its visualisation of alteration in dependence of time as a DTM. Thus far data acquisition has been carried out by applying tacheometry and RTK-GNSS surveys. Both measurement procedures are quite time-consuming and describe only a rough approximation of the ground surface due to the discretisation during the survey. Classical aerial photogrammetry carried out with aeroplanes possesses the advantage of laminar data acquisition of a whole area. Due to high operational costs this method is only rarely or not at all brought into operation. By means of UAV photogrammetry the advantage of extensive data acquisition should be utilised at a low cost. The application area is a landfill covering about $25000 \mathrm{~m}^{2}$.
Prior to the work in the field an aerial flight plan has been derived. The aerial survey should occur at an altitude of $50 \mathrm{~m}$ with longitudinal overlap of $60 \%$ while lateral coverage extends $40 \%$. On-site eight reference points have been determined by deploying a RTK-GNSS survey on the outer margins of the landfill. Subsequently the Oktokopter has been equipped with a camera. From 600 captured images 300 have been chosen for further processing. Due to this large amount of images the automatic generation of the 3D point cloud took 28 hours. As a result a point cloud with 4.8 million points was computed which leads to an average density of 192 points per $\mathrm{m}^{2}$. Figure 6 shows the calculated point cloud. After georeferencing of the point cloud the computation of a triangulated mesh and contour lines was carried out. The outcome of this computation was a DTM as well as a colour coded contour plot.

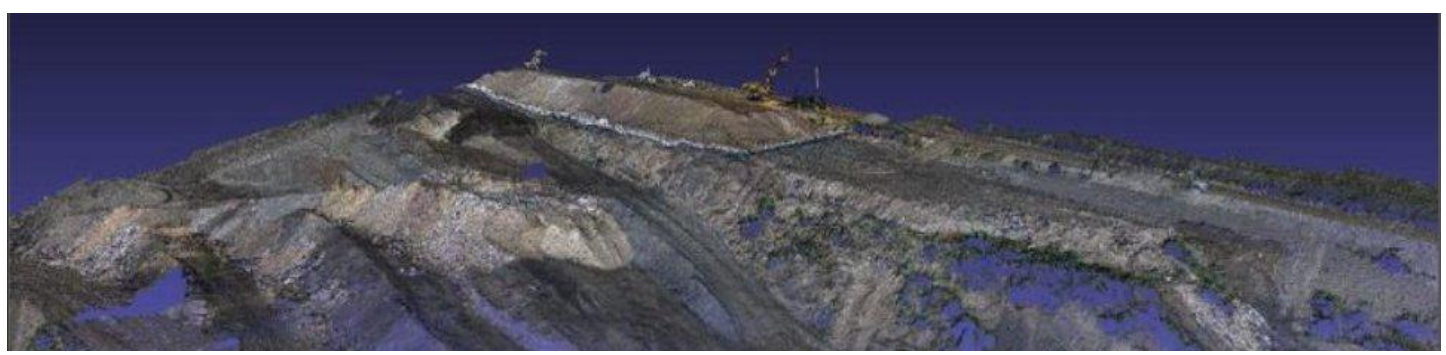

Figure 6. Point cloud of the landfill generated with PMVS2

\section{CONCLUSION}

A workflow for generating 3D point clouds from digital imagery captured by a low-cost UAV was presented. A comparison of various software products has shown that the point clouds differ in density and completeness. Investigating the absolute accuracy of a georeferenced $3 \mathrm{D}$ point cloud it can be noticed that the deviations are dependent from the applied software. However, an absolute point deviation of ca. $20 \mathrm{~cm}$ makes UAV photogrammetry applicable for topographic surveys. A successful application on example of a landfill survey was presented.

\section{REFERENCES}

Eisenbeiß, H., 2009. UAV Photogrammetry. Dissertation. Institut für Geodäsie und Photogrammetrie an der ETH Zürich, Mitteilungen Nr. 105.
Furukawa, Y., Curless, B., Seitz, S.M., Szeliski, R., 2010. Towards Internet-scale multi-view stereo. IEEE Conference on Computer Vision and Pattern Recognition (CVPR), pp. 14341441.

Furukawa, Y., Ponce, J., 2010. Accurate, Dense, and Robust Multiview Stereopsis. IEEE Transactions on Pattern Analysis and Machine Intelligence, Volume 32 Issue 8, pp. 1362-1376.

Lowe, D. (2004). Distinctive image features from scaleinvariant keypoints. International Journal of Computer Vision, 60(2), 91-110.

Snavely, N., Seitz, S.M., Szeliski, R., 2007. Modeling the World from Internet Photo Collections. International Journal of Computer Vision, Volume 80, Number 2, pp. 189-210. 